\title{
TWISTED QUANTUM DOUBLES
}

\section{DAIJIRO FUKUDA and KEN'ICHI KUGA}

\author{
Received 26 February 2003
}

Dedicated to Professor Yukio Matsumoto's sixtieth birthday

\begin{abstract}
Using diagrammatic pictures of tensor contractions, we consider a Hopf algebra $\left(A_{\text {op }} \otimes^{\mathscr{R}_{\lambda}}\right.$ $\left.A^{*}\right)^{*}$ twisted by an element $\mathscr{R}_{\lambda} \in A^{*} \otimes A_{\text {op }}$ corresponding to a Hopf algebra morphism $\lambda$ : $A \rightarrow A$. We show that this Hopf algebra is quasitriangular with the universal $R$-matrix coming from $\mathscr{R}_{\lambda}$ when $\lambda^{2}=\mathrm{id}_{A}$, generalizing the quantum double construction which corresponds to the case $\lambda=\operatorname{id}_{A}$.
\end{abstract}

2000 Mathematics Subject Classification: 17B37, 16W30, 81R50.

1. Introduction. Let $A$ be a Hopf algebra over a field $k$, and $\mathscr{R}$ the canonical element in $A \otimes_{k} A^{*}$ corresponding to the identity map $\operatorname{id}_{A}: A \rightarrow A$. Let $A^{*}$ be the dual Hopf algebra of $A$, and $A_{\text {op }}$ the Hopf algebra with the multiplication opposite to that of $A$, that is, if $\mu_{A}: A \otimes A \rightarrow A$ and $S_{A}: A \rightarrow A$ are the multiplication and the antipode of $A$, then $A_{\mathrm{op}}$ has the multiplication $\mu_{A_{\mathrm{op}}}$ and the antipode $S_{A_{\mathrm{op}}}$ defined by $\mu_{A_{\mathrm{op}}}(x \otimes y):=\mu_{A}(y \otimes x)$ and $S_{A_{\mathrm{op}}}(x):=S_{A}^{-1}(x)$ for $x, y \in A$, while other structures are the same. Then one can define a Hopf algebra structure on the tensor product $A^{*} \otimes_{k} A_{\mathrm{op}}$ by defining the comultiplication and the antipode twisted by $\mathscr{R}$ as

$$
\begin{gathered}
\Delta(\xi \otimes x)=\mathscr{R}_{41} \Delta_{13}^{A^{*}}(\xi) \Delta_{24}^{A_{\mathrm{op}}}(x) \mathscr{R}_{41}^{-1}, \\
S(\xi \otimes x)=\mathscr{R}_{21}^{-1}\left(S_{A^{*}}(\xi) \otimes S_{A_{\mathrm{op}}}(x)\right) \mathscr{R}_{21} .
\end{gathered}
$$

Other structures, that is, multiplication, unit, and counit, are the usual ones on the tensor product.

Let $D(A)=\left(A^{*} \otimes A_{\mathrm{op}}\right)^{*}$ and let $R \in D(A) \otimes D(A)$ be the image of $\mathscr{R}$ under the mapping $A_{\text {op }} \otimes A^{*} \rightarrow A_{\text {op }} \otimes 1_{A^{*}} \otimes 1_{A} \otimes A_{\text {op }} \rightarrow D(A) \otimes D(A)$. Then $D(A)$ turns out to be a quasitriangular Hopf algebra with the universal $R$-matrix $R$.

This is the quantum double construction due to Drinfel'd [2], and it provides an explicit solution to the quantum Yang-Baxter equation (QYBE). The important example is the universal $R$-matrix of the quantized universal enveloping algebra $U_{h}(\mathfrak{g})$ which is obtained as the homomorphic image of the universal $R$-matrix of the quantum double $D\left(U_{h}^{\geq 0}(\mathfrak{g})\right)$ (see [2, 4]). To avoid subtleties concerning Hopf algebra duals or topologies on tensor products for infinite-dimensional algebras and make the basic argument clear, we assume finite dimensionality of Hopf algebras throughout this paper. However, the necessary modifications for some infinite-dimensional cases are standard and will be treated in a subsequent paper. 
Although a QYBE a priori has infinitely many solutions, certain uniqueness results can be obtained for $R$-matrix of the form $\Sigma e^{i} \otimes e_{i}$ as in the quantum double construction (see $[3,4]$ ). Hence, to generalize the quantum double construction, we want to consider general elements $\mathscr{R}_{\lambda}$ in $A_{\mathrm{op}} \otimes_{k} A^{*}$ corresponding to maps $\lambda: A \rightarrow A$. Following the same construction as above using $\mathscr{R}_{\lambda}$ in place of $\mathscr{R}$, we arrive at $D_{\lambda}(A)=\left(A_{\mathrm{op}} \otimes^{\mathscr{R}_{\lambda}} A^{*}\right)^{*}$ which unfortunately fails to be a quasitriangular Hopf algebra for general $\lambda$. The purpose of this paper is to show the following theorem.

THEOREM 1.1. If $\lambda$ is a bialgebra endomorphism of $A$ such that $\lambda \circ \lambda=\mathrm{id}_{A}$, then $D_{\lambda}(A)=\left(A_{\mathrm{op}} \otimes^{\mathscr{R}_{\lambda}} A^{*}\right)^{*}$ becomes a quasitriangular Hopf algebra with the universal $R$ matrix given by the image of $\mathscr{R}_{\lambda}$ under the mapping $A_{\mathrm{op}} \otimes A^{*} \rightarrow A_{\mathrm{op}} \otimes 1_{A^{*}} \otimes 1_{A} \otimes A_{\mathrm{op}} \rightarrow$ $D(A) \otimes D(A)$.

For a Hopf algebra example, if $A$ is commutative and cocommutative, then the antipode $S_{A}: A \rightarrow A$ becomes a bialgebra isomorphism satisfying $S_{A} \circ S_{A}=\mathrm{id}_{A}$. Another class of examples of such order-2 bialgebra automorphisms may come from the Weyl group acting as a group of reflections on the Cartan subalgebra of a simple Lie group $\mathfrak{g}$. In fact, this action lifts to an action of a finite covering of the Weyl group on the universal enveloping algebra $U(\mathfrak{g})$ as a group of automorphisms.

The proof would look fairly complicated in the usual formalism of tensor manipulations because of the abundance of tensor components and indices involved. So, we want to present the proof using diagrams representing contraction of indices of various tensors. These diagrams will make the proof more visible and understandable since duality and other symmetry properties of the arguments become rather obvious. So, we begin with writing Hopf algebra structures using these diagrams.

2. Diagrams representing Hopf algebra structures. We write fixed basis elements of $A$ and the dual basis elements of $A^{*}$ as $\downarrow$ and $\uparrow$ respectively. Hence, $A$ has a basis $\left\{e^{i}=\stackrel{(i)}{\downarrow}\right\}_{i \in I}$ and $A^{*}$ has the dual basis $\left\{e_{j}=\underset{(j)}{\}_{i \in I}}:\langle\stackrel{(i)}{\perp},\rangle_{(j)}\right\rangle=\delta_{j}^{i}$.

The multiplication $\mu_{A}: A \otimes A \rightarrow A$ and the unit $\iota=\iota_{A}: k \rightarrow A$ are expressed as $\mu_{A}(\stackrel{(i)}{\square} \stackrel{(j)}{\downarrow})=\underbrace{(i)(j)}$ and $\iota(1)=1_{A}=\sum_{i} \iota_{i} e^{i}=\stackrel{l}{l}$. Hence, the diagram $\underbrace{(i)(j)}_{(k)}$ represents the coefficient $\mu_{k}^{i j}$ in $\mu_{A}\left(e^{i} \otimes e^{j}\right)=\sum_{k} \mu_{k}^{i j} e^{k}$.

The comultiplication $\Delta^{A}: A \rightarrow A \otimes A$ and the counit $\epsilon=\epsilon^{A}: A \rightarrow k$ are expressed as

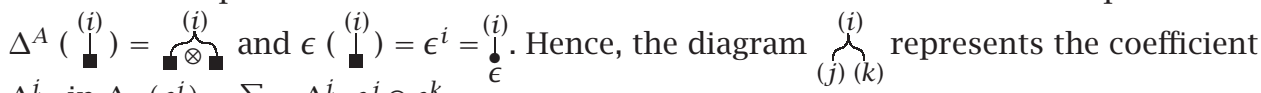
$\Delta_{j k}^{i}$ in $\Delta_{A}\left(e^{i}\right)=\sum_{j, k} \Delta_{j k}^{i} e^{j} \otimes e^{k}$.

Associativity and unitality, coassociativity and counitality may be expressed in a form where duality is obvious:

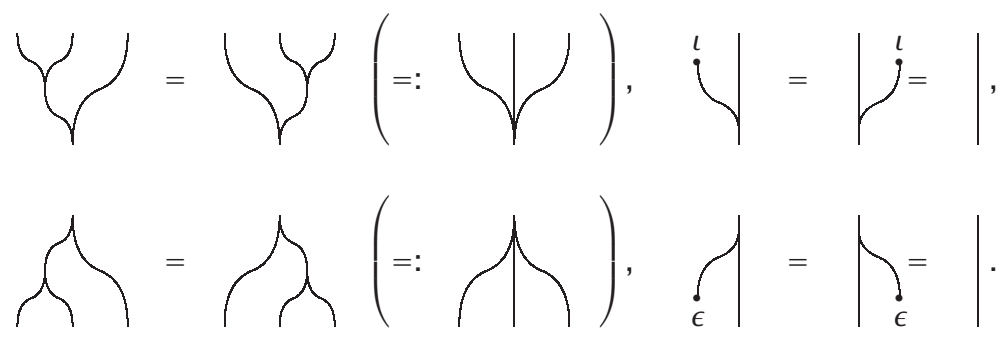


Compatibility of algebra and coalgebra structures is

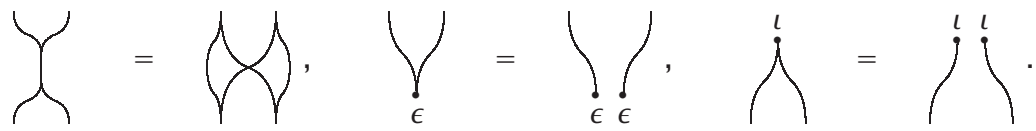

The antipode $S_{A}: A \rightarrow A$ is written as $S_{\uparrow}$ and satisfies<smiles>C1CCCC1</smiles>

where $S$ is an antiautomorphism of the Hopf algebra $A$ :

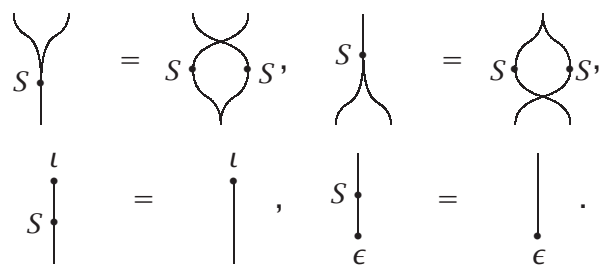

In general, a linear map $f: A \rightarrow A$ is written as $f(\stackrel{(i)}{!})=f \stackrel{(i)}{\dagger}$, expressing $f\left(e^{i}\right)=\sum_{i} f_{j}^{i} e^{j}$. A linear map $f$ is an algebra homomorphism when

$$
f=f \cdot \rho f, \quad f^{\natural}=\stackrel{\iota}{\dagger} .
$$

A linear map $f$ is a coalgebra homomorphism when

$$
\left.f \nmid=\oint_{f}, \quad f \cdot f_{\epsilon}=\right\rfloor_{\epsilon} .
$$

Note that related Hopf algebras $A^{*}, A_{\mathrm{op}}, A^{\mathrm{op}}$, and so forth can be expressed by using the same components of the diagram for $A$. For example, the multiplication $\mu_{A^{*}}$ of the dual Hopf algebra $A^{*}$ is the transpose of the comultiplication $\Delta^{A}$ of $A$ which is

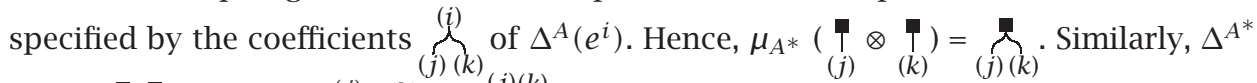

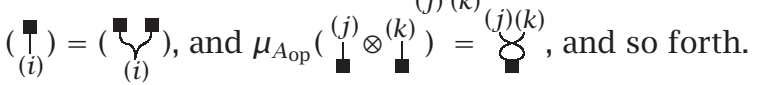

3. Proof of the theorem. Let $\lambda: A \rightarrow A$ be a bialgebra endomorphism and let $\mathscr{R}_{\lambda} \in$ $A \otimes A^{*}$ be the element corresponding to $\lambda: \mathscr{R}_{\lambda}=\sum \lambda_{i}^{j} e^{i} \otimes e_{j}$. Diagrammatically, $\mathscr{R}_{\lambda}=$ $\lambda \otimes \varphi$ 
LEMmA 3.1. The element $\mathscr{R}_{\lambda}$ is an invertible element of $A_{\mathrm{op}} \otimes A^{*}$ satisfying the following relations:

$$
\begin{gathered}
\left(\mathrm{id} \otimes \Delta^{A^{*}}\right)\left(\mathscr{R}_{\lambda}\right)=\left(\mathscr{R}_{\lambda}\right)_{13}\left(\mathscr{R}_{\lambda}\right)_{12}, \quad\left(\Delta^{A_{\mathrm{op}}} \otimes \mathrm{id}\right)\left(\mathscr{R}_{\lambda}\right)=\left(\mathscr{R}_{\lambda}\right)_{13}\left(\mathscr{R}_{\lambda}\right)_{23}, \\
\left(\mathrm{id} \otimes S^{A^{*}-1}\right)\left(\mathscr{R}_{\lambda}\right)=\mathscr{R}_{\lambda}^{-1}, \quad\left(S^{A_{\mathrm{op}}} \otimes \mathrm{id}\right)\left(\mathscr{R}_{\lambda}\right)=\mathscr{R}_{\lambda}^{-1} .
\end{gathered}
$$

\section{ProOF.}

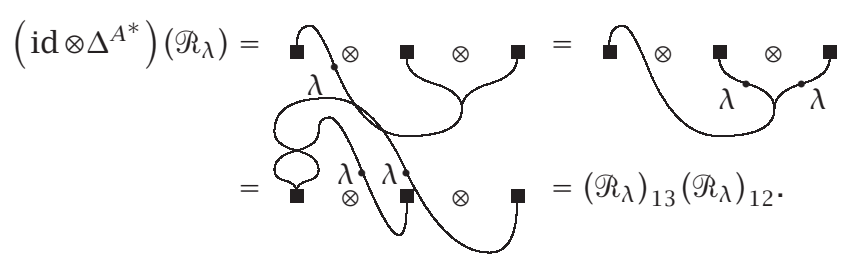

This proves the first equality.

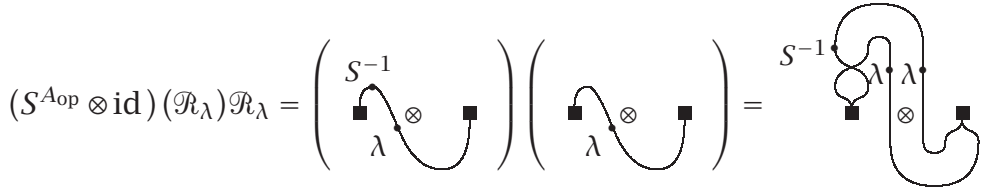

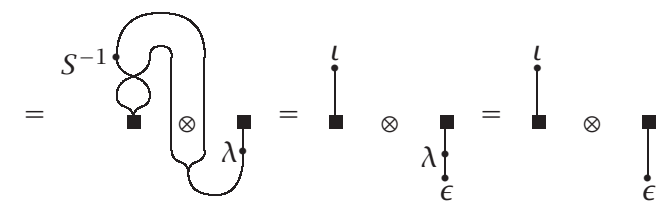

$$
\begin{aligned}
& =1_{A_{\mathrm{op} \otimes A^{*}}} \text {. }
\end{aligned}
$$

Here we used $S^{-1} \bigotimes=\epsilon_{\iota} !$.

The remaining equalities may be similarly verified.

On the tensor product $A^{*} \otimes_{k} A_{\text {op }}$ we define the multiplication $M$, the comultiplication $\Delta_{\lambda}$, the unit $\mathscr{I}$, the counit $\mathscr{E}$, and the antipode $S_{\lambda}$ as follows:

$$
\begin{gathered}
\mathcal{M}((\xi \otimes x) \otimes(\eta \otimes y))=(\xi \otimes x)(\eta \otimes y)=\mu_{A^{*}}(\xi \otimes x) \otimes \mu_{A_{\mathrm{op}}}(\eta \otimes y), \\
\Delta_{\lambda}(\xi \otimes x)=\left(\mathscr{R}_{\lambda}\right)_{41} \Delta_{13}^{A^{*}}(\xi) \Delta_{24}^{A_{\mathrm{op}}}(x)\left(\mathscr{R}_{\lambda}\right)_{41}^{-1}, \\
\mathscr{T}(1)=\epsilon^{A} \otimes \iota_{A}=1_{A^{*} \otimes A_{\mathrm{op}}}, \quad \mathscr{E}(\xi \otimes x)=\xi\left(1_{A}\right) \epsilon^{A}(x), \\
S_{\lambda}(\xi \otimes x)=\left(\mathscr{R}_{\lambda}^{-1}\right)_{21}\left(S_{A^{*}}(\xi) \otimes S_{A_{\mathrm{op}}}(x)\right)\left(\mathscr{R}_{\lambda}\right)_{21}, \\
\left(\text { for } \xi, \eta \in A^{*}, x, y \in A_{\mathrm{op}}\right) .
\end{gathered}
$$

Hence, the comultiplication and the antipode are twisted by $\lambda$, while other structures are the usual ones on the tensor product. 
As $\mathscr{R}_{\lambda}^{-1}=S_{\lambda}^{-1} \otimes$ from Lemma 3.1, the twisted comultiplication may be written diagrammatically as

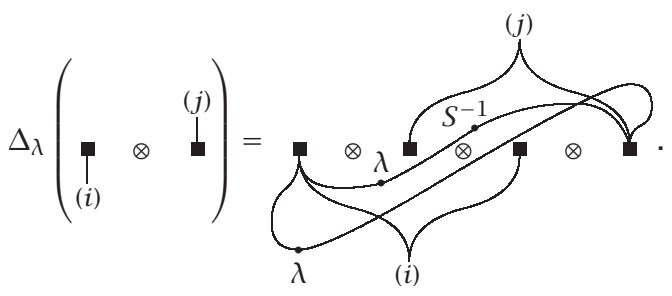

Similarly,

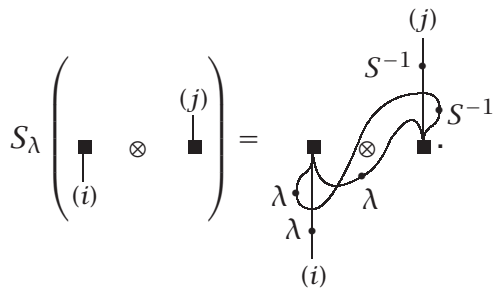

Proposition 3.2 (see $[1,2,4])$. The system $\left(A^{*} \otimes A_{\mathrm{op}}, \mathcal{M}, \mathscr{\Phi}, \Delta_{\lambda}, \mathscr{E}, S_{\lambda}\right)$ is a Hopf algebra.

Proof. This is a standard fact. More generally, if an invertible element $\mathscr{R} \in C \otimes B$ satisfies the conclusion of Lemma 3.1, namely,

$$
\begin{gathered}
\left(\operatorname{id} \otimes \Delta^{B}\right)(\mathscr{R})=\mathscr{R}_{13} \mathscr{R}_{12}, \quad\left(\Delta^{C} \otimes \text { id }\right)(\mathscr{R})=\mathscr{R}_{13} \mathscr{R}_{23}, \\
\left(\operatorname{id} \otimes\left(S^{B}\right)^{-1}\right)(\mathscr{R})=\mathscr{R}^{-1}, \quad\left(S^{C} \otimes \text { id }\right)(\mathscr{R})=\mathscr{R}^{-1},
\end{gathered}
$$

then $B \otimes C$ becomes a Hopf algebra with the structures defined as above. The verification of Hopf algebra conditions may be a little tedious but can be done without difficulty. Hence we omit it.

Let $D_{\lambda}(A)=\left(\left(A^{*} \otimes^{\mathscr{R}_{\lambda}} A_{\mathrm{op}}\right)^{*}, \mu_{D}, \mathscr{I}_{D}, \Delta^{D}, \mathscr{C}^{D}, S_{D}\right)$ be the dual Hopf algebra of $\left(A^{*} \otimes\right.$ $\left.A_{\text {op }}, \mathcal{M}, \mathscr{I}, \Delta_{\lambda}, \mathscr{E}, S_{\lambda}\right)$ and let $R_{\lambda} \in D_{\lambda}(A) \otimes D_{\lambda}(A)$ be the image of $\mathscr{R}_{\lambda} \in A \otimes A^{*}$ under the map $A \otimes A^{*} \rightarrow A \otimes 1_{A}^{*} \otimes 1_{A} \otimes A^{*} \rightarrow D_{\lambda}(A)$. We now assume $\lambda \circ \lambda=\mathrm{id}_{A}$. We want to show that $\left(D_{\lambda}(A), R_{A}\right)$ is quasitriangular, that is, we need to check the following conditions:

$$
\begin{gathered}
\left(\operatorname{id} \otimes \Delta^{D}\right)\left(R_{\lambda}\right)=\left(R_{\lambda}\right)_{13}\left(R_{\lambda}\right)_{12}, \quad\left(\Delta^{D} \otimes \mathrm{id}\right)\left(R_{\lambda}\right)=\left(R_{\lambda}\right)_{13}\left(R_{\lambda}\right)_{23}, \\
R_{\lambda} \Delta^{D}(x)=\Delta^{D_{\mathrm{op}}}(x) R_{\lambda} \quad \text { for } x \in D_{\lambda}(A),
\end{gathered}
$$

where $\Delta^{D_{\text {op }}}$ is the opposite of the comultiplication $\Delta^{D}$.

We will give a proof for the last equality. The first two equalities can be verified easily. 
Noting that the multiplication $\mu_{D}$ of $D_{\lambda}(A)$ is the dual of the comultiplication $\Delta_{\lambda}$ of $A^{*} \otimes A_{\mathrm{op}}$, whose diagrammatic description is given at the end of Section 2 , we see that

$$
\Delta^{D_{\mathrm{op}}}\left(\begin{array}{ccc}
(a) & & \\
\downarrow & \otimes & \varphi \\
& & (b)
\end{array}\right) R_{\lambda}
$$
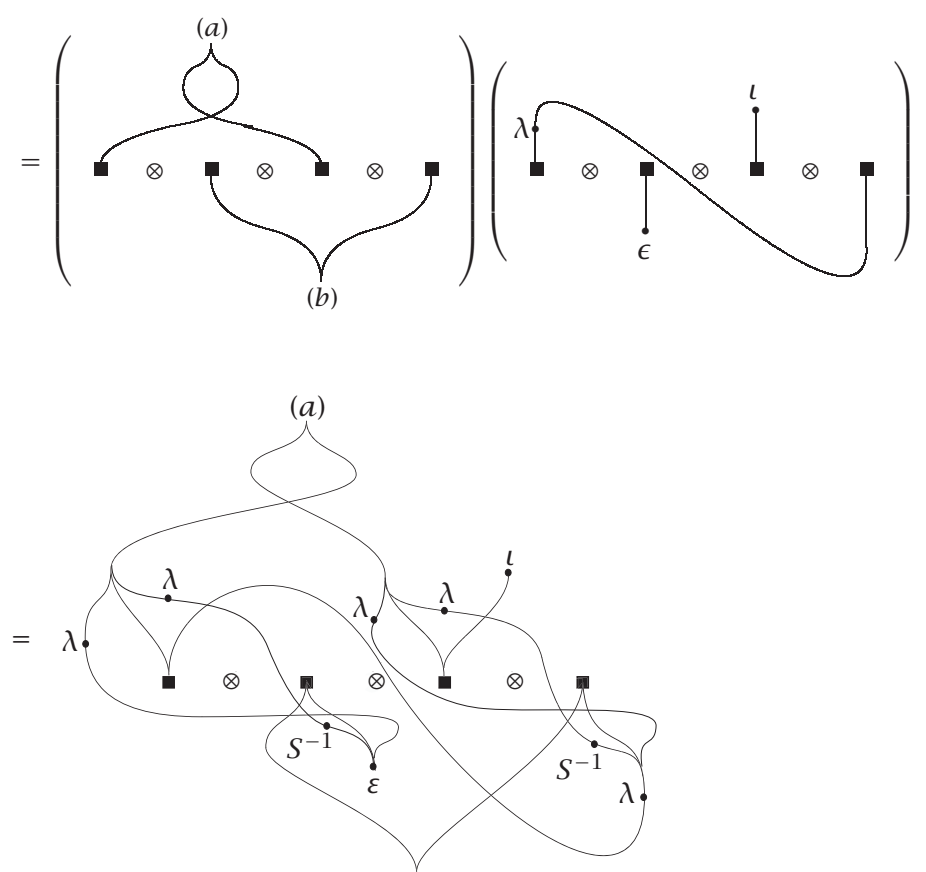

(b)

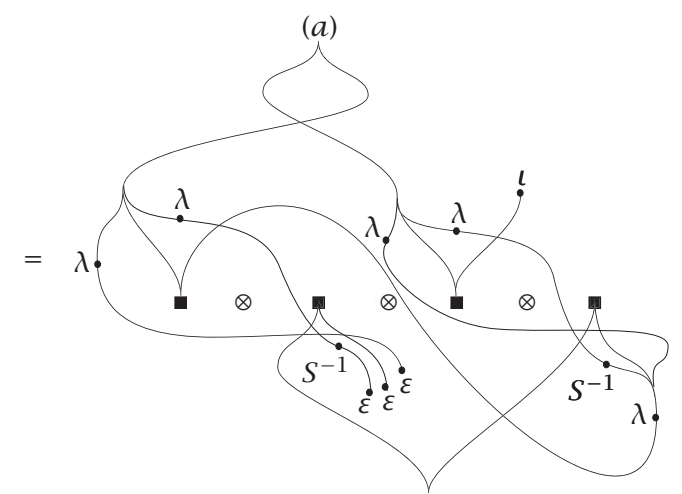

(b)

(by the counitality of the multiplication) 

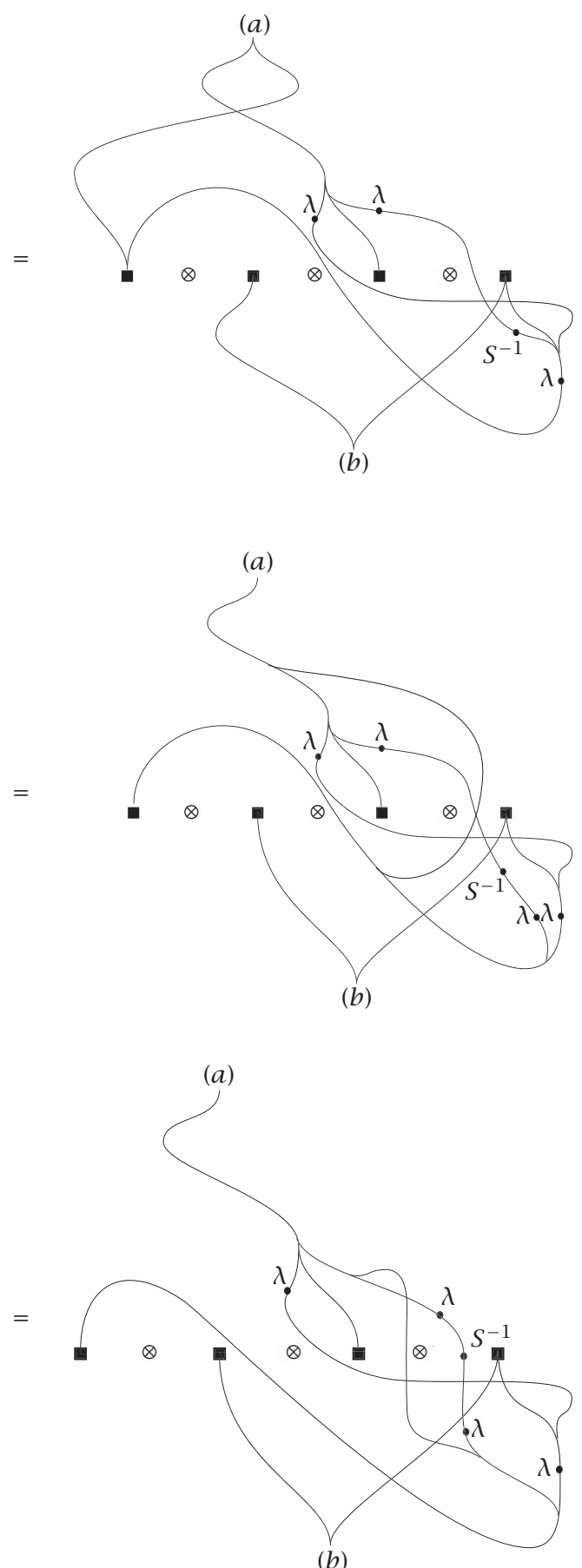

(by associativity and coassociativity) 


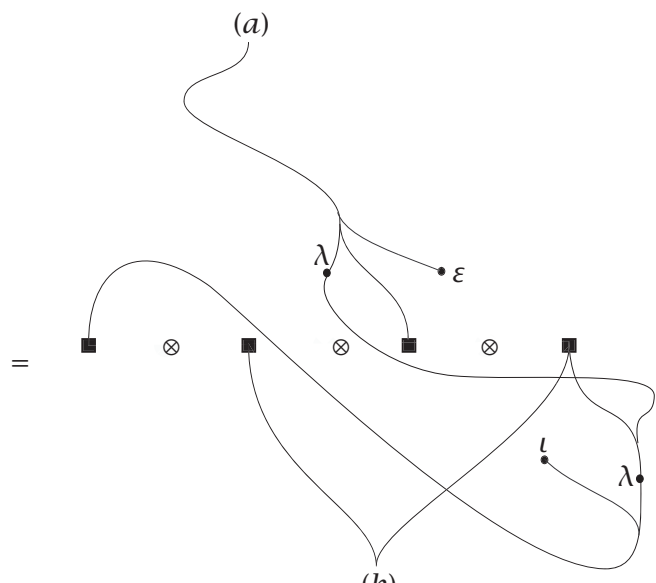

(b)
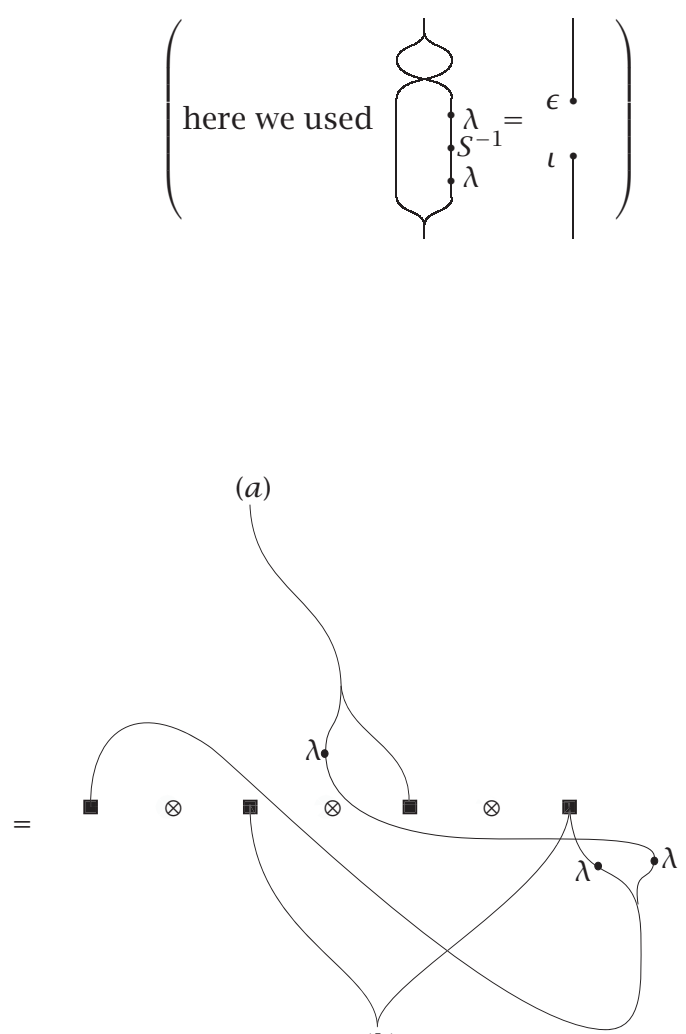

(b) 

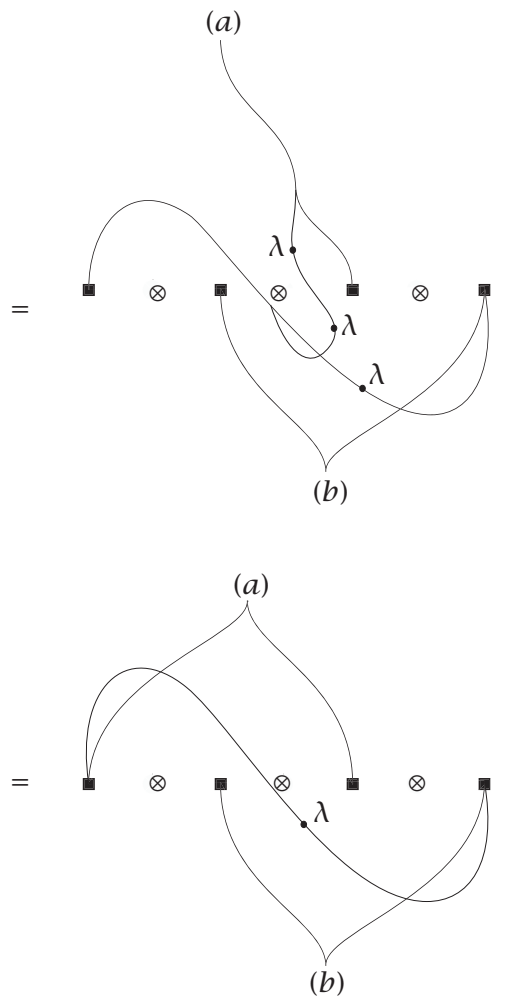

(by the hypothesis $\lambda^{2}=\mathrm{id}$ ).

The other side of the desired equality is diagrammatically

$$
R_{\lambda} \Delta^{D}\left(\begin{array}{ccc}
(a) & & \\
\downarrow & \otimes & \square \\
& & (b)
\end{array}\right)
$$

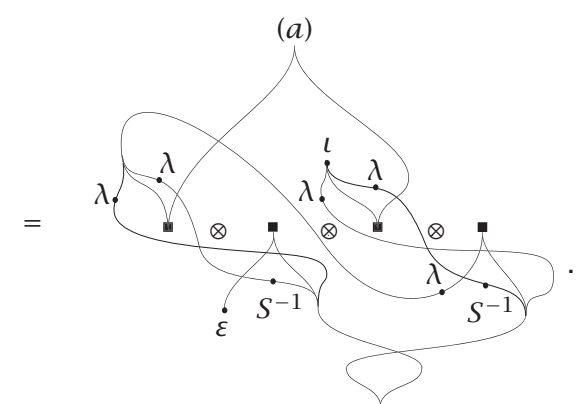

(b) 
From the symmetry between this diagram and the first one and using the duality between the associativity and coassociativity, and so forth, we can modify this diagram in exactly the same way and obtain the same final diagram as above. This completes the proof of Theorem 1.1.

\section{REFERENCES}

[1] V. Chari and A. Pressley, A Guide to Quantum Groups, Cambridge University Press, Cambridge, 1994.

[2] V. G. Drinfel'd, Quantum groups, Proceedings of the International Congress of Mathematicians, Vol. 1, 2 (Berkeley, Calif, 1986), American Mathematical Society, Rhode Island, 1987, pp. 798-820.

[3] S. M. Khoroshkin and V. N. Tolstoy, The uniqueness theorem for the universal R-matrix, Lett. Math. Phys. 24 (1992), no. 3, 231-244.

[4] S. Majid, Physics for algebraists: noncommutative and noncocommutative Hopf algebras by a bicrossproduct construction, J. Algebra 130 (1990), no. 1, 17-64.

Daijiro Fukuda: Department of Mathematics and Informatics, Faculty of Science, Chiba University, Chiba 263-8522, Japan

E-mail address: dfukuda@g.math.s.chiba-u.ac.jp

Ken'ichi Kuga: Department of Mathematics and Informatics, Faculty of Science, Chiba University, Chiba 263-8522, Japan

E-mail address: kuga@math.s.chiba-u.ac.jp 


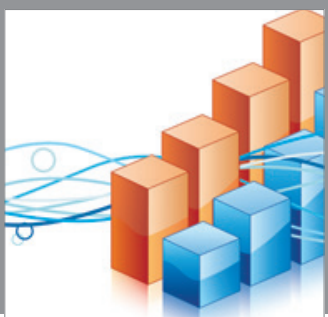

Advances in

Operations Research

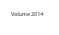

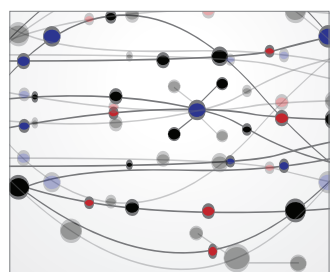

\section{The Scientific} World Journal
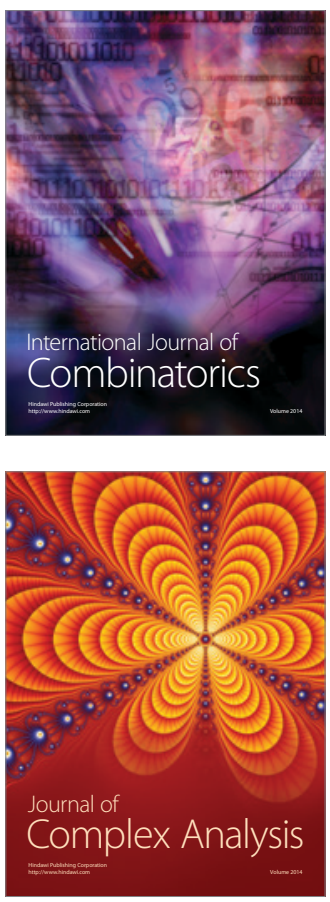

International Journal of

Mathematics and

Mathematical

Sciences
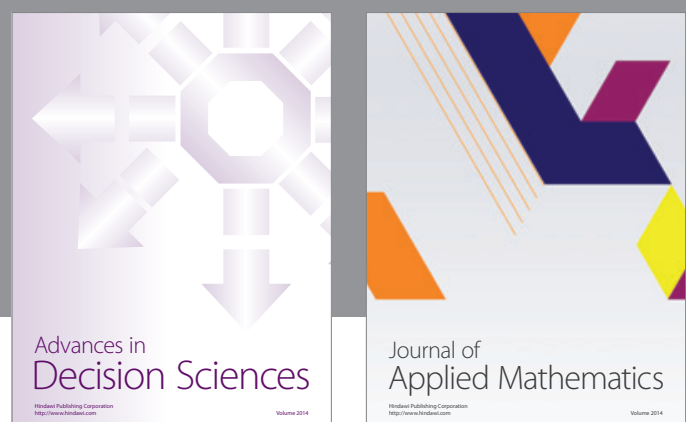

Journal of

Applied Mathematics
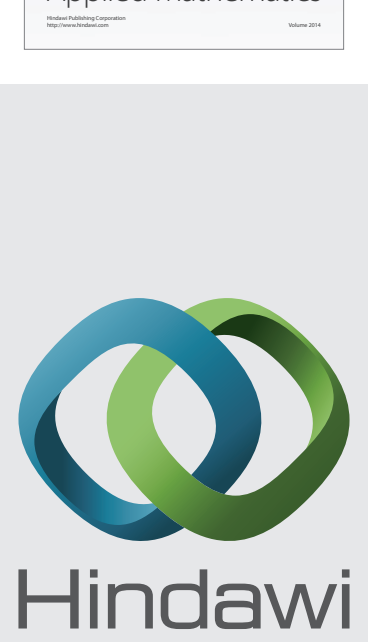

Submit your manuscripts at http://www.hindawi.com
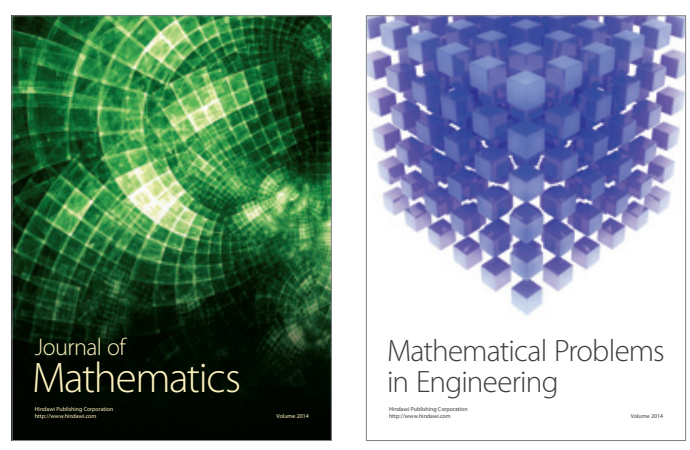

Mathematical Problems in Engineering
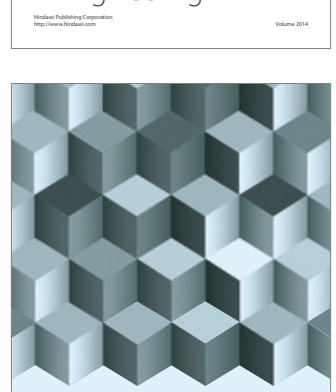

Journal of

Function Spaces
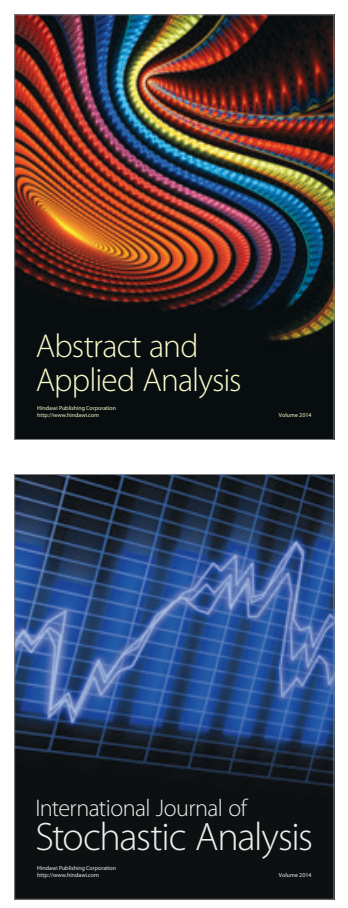

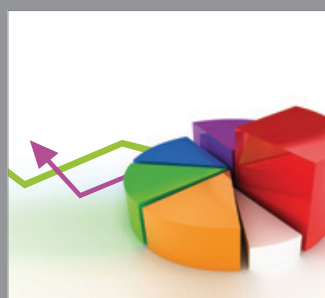

ournal of

Probability and Statistics

Promensencen
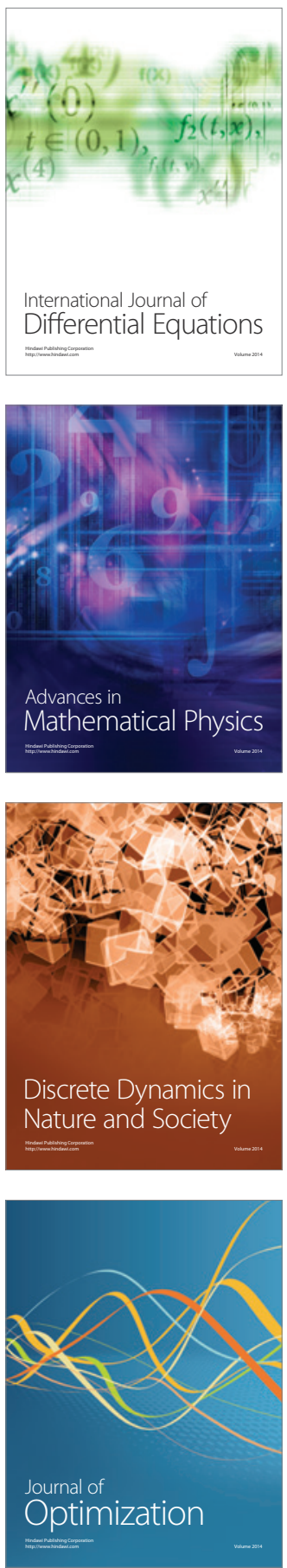